\title{
Identification of muskmelon seed variety using hyperspectral imaging technology combined with machine learning
}

\author{
Kunlan Luo ${ }^{1}$, Wenchao Ye ${ }^{1}$, Yuhao Diao ${ }^{1}$, Jing Zhao ${ }^{1,2,3^{*}}$, Wanjun Chen ${ }^{1}$, \\ Houcheng Liu', Yongbing Long ${ }^{1,2,3^{*}}$ \\ (1. School of Electronic Engineering, South China Agricultural University, Guangzhou 510642, China; \\ 2. Lingnan Modern Agricultural Science and Technology Guangdong Laboratory, Guangzhou 510642, China; \\ 3. National Center for International Collaboration Research on Precision Agricultural Aviation Pesticides Spraying Technology (NPAAC), \\ South China Agricultural University, Guangzhou, 510642, China; \\ 4. College of Horticulture, South China Agricultural University, Guangzhou 510642, China)
}

\begin{abstract}
Liquid crystal tunable filter (LCTF) based on hyperspectral imaging technology combined with machine learning is developed to identify muskmelon seed variety rapidly and non-destructively. LCTF-based hyperspectral imaging system equipped with a cold ring LED source is constructed to acquire the reflectance spectra of the muskmelon seeds. Discriminating models based on support vector machine (SVM), linear discriminant analysis (LDA), and convolutional neural network (CNN) are then established to identify the seed variety with reflectance spectra as input. It is found that the LDA model achieved the highest classification accuracy of $100 \%$ for the test set while a relatively low value of $96 \%$ and $83 \%$ was obtained for the SVM and CNN model respectively. To improve classification accuracy of the model, data preprocessing (Savitzky-Golay smoothing and multiple scattering correction) and spectral feature extraction algorithm (successive projections algorithm and principal component analysis) were employed to treat the reflectance data. With these treatments, the classification accuracy of the test set was improved to the highest value of over 99\% for the SVM model and 86\% for the CNN model. The results showed that the LCTF-based hyperspectral imaging technology combined with machine learning was feasible to identify the muskmelon seed variety.
\end{abstract}

Keywords: hyperspectral imaging; liquid crystal tunable filter; variety identification; muskmelon seed; support vector machine; linear discriminant analysis; convolution neural network

DOI: $10.33440 /$ j.ijpaa.20200303.93

Citation: Luo K L, Ye W C, Diao Y H, Zhao J, Chen W J, Liu H C, Long Y B. Identification of muskmelon seed variety using hyperspectral imaging technology combined with machine learning. Int J Precis Agric Aviat, 2020; 3(3): 14-20.

\section{Introduction}

Muskmelon is one of the most important fruit in the world due to the advantages of health enhancement, short cultivation cycle, and remarkable economic effects ${ }^{[1]}$. Muskmelon variety, seed quality, environmental and nutritional factors are key elements determining the yield and quality of muskmelon, which are directedly related to the interests of fruit farmers and consumers. Therefore, the variety, purity and quality are critical in planting and growing of the muskmelons. The variety of the muskmelon seeds can be identified by observing the seed appearance such as seed size, color, shape and texture, etc ${ }^{[2]}$. Chemical methods such as

Received date: 2020-07-29 Accepted date: 2020-09-17

Biographies: Kunlan Luo, master candidate, research interests: application of hyperspectral imaging technology in precision agriculture; 554576830@qq.com; Wenchao Ye, master candidate, research interests: precision agricultural technology.1299870652@qq.com; Yuhao Diao, master candidate, research interests: machine vision. 2228794622@qq.com; Wanjun Chen, research interests: application of hyperspectral imaging technology in precision agriculture.1134881509@qq.com. Houcheng Liu, PhD, Professor, research interests: protected horticulture production, Email: liuhch@scau.edu.cn;

* Corresponding author: Yongbing Long, PHD, professor, research interests: machine vision, artificial intelligence in precision agriculture. Mailing Address: School of Electronic Engineering, South China Agricultural University, Guangzhou, China, Email: yongbinglong@126.com; Jing Zhao, PHD, associate professor, research interests: machine vision, artificial intelligence in precision agriculture, Mailing Address: School of Electronic Engineering, South China Agricultural University, Guangzhou, China. Email: edithzj@126.com. high-performance liquid chromatography and gas chromatography-mass spectrometry are also commonly used to identify seed varieties by detecting the internal qualities such as protein, starch and aroma ${ }^{[3,4]}$. These methods, however, have the disadvantages of seed damage, time-consuming and high cost, and can only be applied on a small number of samples. Therefore, it is critical to develop rapid and non-destructive testing technology to identify the variety of muskmelon seeds.

With the development of machine learning, many kinds of non-destructive technology were proposed for seeds identification. Xianxi Liu group analyzed the geometric and color feature parameters of RGB images of corn seeds based on machine learning. Four kinds of corn seeds were classified, and the accuracy rate reach to $95 \%{ }^{[5]}$. Jia group combined near-infrared spectrum and SVM algorithm to identify four kinds of corn seeds, and the accuracy rate reach to $97.5 \%{ }^{[6]}$. Cuilin Li group proposed the fluorescence reflectance method to test muskmelon seeds, which proved the possibility of identification muskmelon seeds with non-destructive technique combined with appropriate machine learning algorithm ${ }^{[7]}$.

Hyperspectral imaging technology has been widely used in detecting and monitoring of agricultural production since it can obtain both the internal and external information of the sample rapidly and non-destructively ${ }^{[8-25]}$. It has been verified in previous research that the hyperspectral imaging technology is an efficient approach to detect the growth information of crops $^{[8,9]}$, predict maturity and yield, track pests and diseases ${ }^{[11,12]}$, detect the soil 
nutrient ${ }^{[13]}$, meat quality ${ }^{[14]}$ and pesticide residue ${ }^{[15-17]}$. At present, the technology is also applied to identify seed variety and detect the vigor, component and purity of the seeds ${ }^{[18-25]}$. The hyperspectral imaging system used in these researches mainly consists of imaging spectrograph based on optical gratings and hot tungsten halogen lamps. In this type of system, however, the samples are required to move in a direction perpendicular to scanning-line and may be heated and damaged by the hot light source.

This paper aims to propose a liquid crystal tunable filter (LCTF) based on hyperspectral imaging technology equipped with a cold ring LED source to identify the muskmelon seed variety. In this system, the seed samples are neither required to be moved nor to be heated due to the use of LCTF and cold LED source. The LCTF-based hyperspectral imaging system was constructed to obtain reflectance data of 1100 muskmelon samples of eleven varieties. With the reflectance data, discriminating models based on support vector machine (SVM), linear discriminant analysis (LDA), and convolutional neural network (CNN) were established to identify the seed varieties. Besides, data preprocessing and spectral feature extraction algorithm were also employed to optimize the accuracy of the discriminating model.

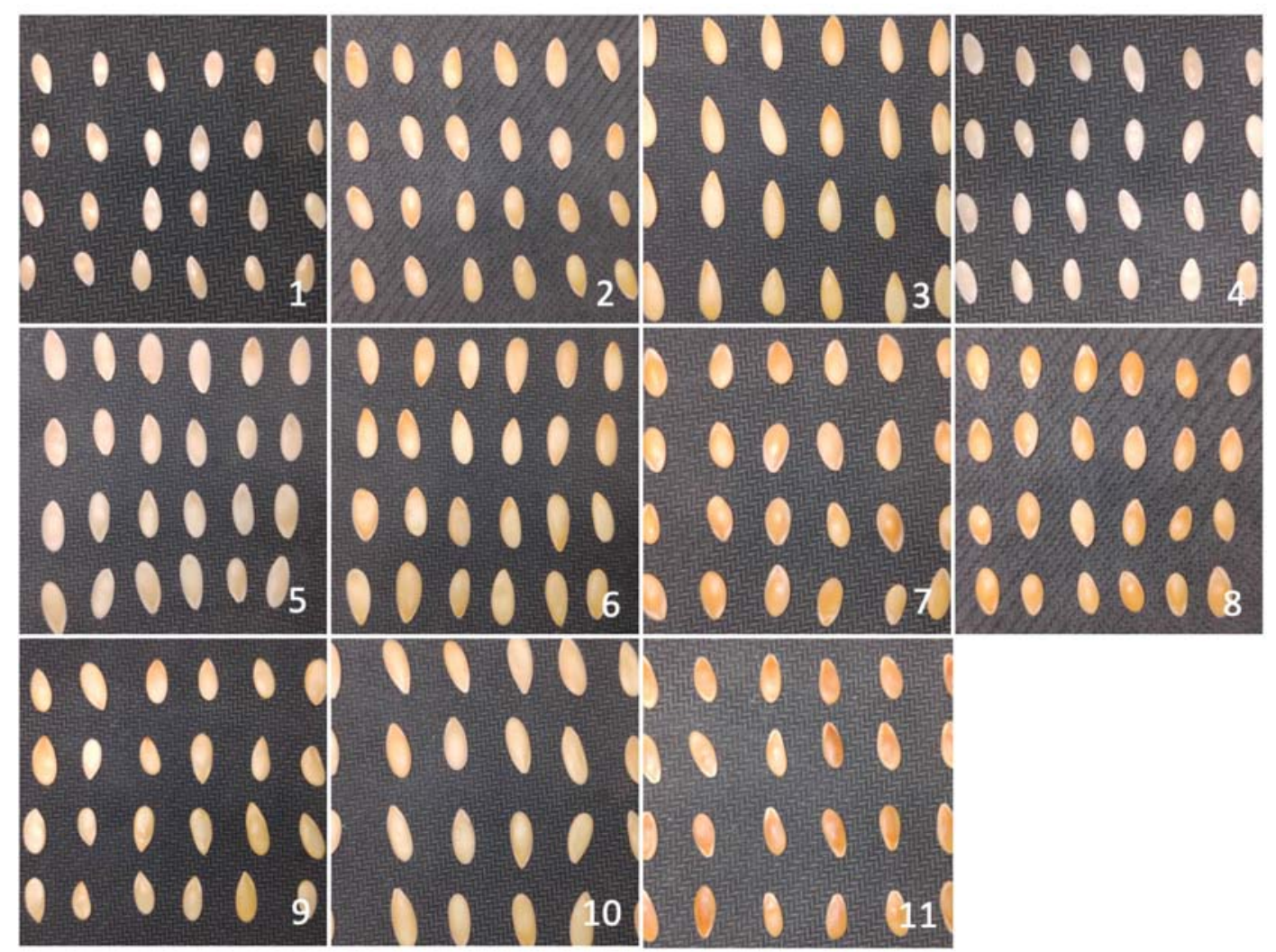

Figure 1 Eleven seed varieties of muskmelon

\section{Material and Method}

\subsection{Material}

Muskmelon seeds used in this paper were purchased from the seed company. The epidermis of seeds is clean and free of foreign matter, and the purity is more than $99 \%$. In this paper, eleven muskmelon seed varieties were used and 100 samples were selected in the experiment for each variety. The appearances of the muskmelon seeds were shown in Figure 1, where Number 1 to 11 respectively correspond to "bairoushabai", "baiyu2000", "hongbaoshiyang”, "hongroushabai”, "lvbaoshiyang”, "meihua”, “meiqi”, “naixiangmiwang”, “kaqihuanghou”, "langchaoA710”, and "sumilong".

\subsection{Hyperspectral imaging system and spectrum acquisition}

LCTF-based hyperspectral imaging system was constructed to collect the reflectance spectra of the muskmelon seeds, as is shown in Figure 2. LCTF-based hyperspectral imaging system mainly consists of hyperspectral imaging camera, LED ring light source, bracket and computer. The hyperspectral imaging camera is mainly formed by LCTF (Varispec VIS, PekinElmer Ltd), charge coupled device (CCD) detector (Im165, Lumenera Ltd.) and a camera lens (Schneider Xenoplan 1.9/35, Schneider Optics Ltd.).
The transmission peak wavelength of the LCTF can be electrically tuned from $400 \mathrm{~nm}$ to $720 \mathrm{~nm}$ at $2 \mathrm{~nm}$ interval. The spatial resolution of the CCD is $1392 \times 1040$. The hyperspectral imaging camera is supported by the bracket (KAISER 5411 RS 2 XA, Photo Warehouse Ltd.) and a cold ring LED light source is used to illuminate the seeds uniformly. A computer is used to control the system with a self-programming software.

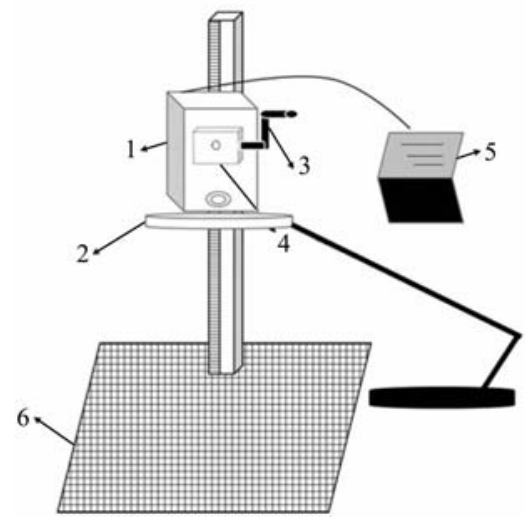

1. Hyperspectral imaging camera 2. LED ring light source 3. Adjusting handle 4. Connecting unit 5. Computer 6. Bracket

Figure 2 Schematic diagram of the hyperspectral imaging system 
In order to reduce the influence of dark current of the CCD detector, the hyperspectral images of the seeds $\left(I_{\text {raw }}\right)$ should be corrected. For this purpose, a standard white reference image ( $I_{\text {white }}$ ) was acquired by using a whiteboard with nearly $100 \%$ reflectance and a dark reference image $\left(I_{\text {black }}\right)$ was acquired by covering the camera lens completely with its opaque cap. The hyperspectral image $(R)$ can be corrected as ${ }^{[16]}$.

$$
R=\frac{I_{\text {raw }}-I_{\text {black }}}{I_{\text {white }}-I_{\text {black }}}
$$

where, $R$ is equivalent to the relative reflectance.

In order to obtain accurate and credible spectral data, Snake active contour segmentation algorithm was used to extract the contour of a single seed ${ }^{[26]}$. All pixel points within the contour of each seed were extracted and the reflectance spectra of all pixels within the contour were averaged and used as the reflectance for a single seed.

\subsection{Spectral preprocessing}

Spectral preprocessing is necessary since the spectral data contain irrelevant information such as noise, stray light, and sample background caused by the instrument and test conditions. To reduce the influence of irrelevant information and highlight the effective information of the spectrum, Savitzky-Golay (SG) smoothing algorithm and multiple scattering correction (MSC) algorithm were employed to preprocess the spectra data ${ }^{[18]}$. The SG smoothing algorithm is an effective approach to reduce the random noise in the spectral data ${ }^{[19]}$. In this research, SG smoothing with 5 points of 3rd order polynomial was used to preprocess the spectral data.

The MSC algorithm can be used to eliminate the effect of scattering and then enhance signal-to-noise ratio. In the MSC algorithm, standard spectrum $\bar{X}$ was first calculated by averaging the spectra of all the seed samples. A univariate linear regression operation $(\chi=\alpha \bar{X}+\beta)$ between the spectrum of each sample $(\chi)$ and the standard spectrum $(\bar{X})$ were then performed to find the linear translation $\alpha$ and tilt offset $\beta$. The corrected spectrum can be expressed as:

$$
\chi_{M S C}=\frac{\chi-\beta}{\alpha}
$$

\subsection{Spectral feature extraction}

The original spectrum of muskmelon contains 68 wavelengths in the range of $450-718 \mathrm{~nm}$ at $4 \mathrm{~nm}$ interval, considering the noise at two edges of spectral range. The high dimensionality of the spectral data and the redundancy of the data may increase the calculation time and affect the accuracy and stability of the model. To circumvent this problem, successive projections algorithm (SPA) and principal component analysis (PCA) were used to extract the spectral feature ${ }^{[18]}$.

SPA is widely used in characteristic wavelength selection in the process of spectral data analysis. The algorithm can find the combination of variables to minimize the redundant information in the spectrum matrix and the co-linearity between the variables. In this method, the number of characteristic wavelengths is determined according to the root mean square error.

PCA converts the original data into a new set of comprehensive variables (principal components). Each principal component is a linear combination of the original variables and the correlation between the variables is eliminated, which helps to improve the stability of the model. In this research, all principal components were sorted according to their contribution rate and the number of selected principal components was determined in order that the cumulative contribution rate reaches $99.99 \%$.

\subsection{Classification algorithm}

\subsubsection{Support vector machine}

Support vector machine (SVM) is a supervised classifier and widely used for spectral data division ${ }^{[22]}$. In the SVM classification algorithm, the original data are mapped to a higher-dimensional space and a hyperplane is constructed to maximize the distance between different classes and minimize the intra-class distance in the same class. Non-linear SVM was applied in this research for data division with radial basis function used as kernel function and the parameters of the model was optimized by grid search algorithm.

\subsubsection{Linear discriminant analysis}

Linear discriminant analysis (LDA) is a classic algorithm that projects high-dimensional pattern samples onto the optimal discriminant vector space to extract the classification information and compress the feature space dimension ${ }^{[20]}$. After projection, the pattern samples have the largest inter-class space and the smallest intra-class distance so that the separability in the new space is maximized.

\subsubsection{Convolutional neural network}

Convolutional neural network (CNN) can learn data features such as peaks and valleys in the spectral curve and then are widely applied to spectral data division ${ }^{[2023]}$. The CNN model used in this paper was one-dimensional Resnet101 with Relu as activation function and Stochastic Gradient Descent (SGD) as loss function, respectively. The batch size and learning rate were respectively set as 30 and 0.005 .

\section{Results and discussion}

\subsection{Hyperspectral characteristics}

The Snake active contour segmentation algorithm was first used to extract the contour of each muskmelon seed sample and the results were shown in Figure $3^{[26]}$. The contour segmentation was performed on a hyperspectral image at the wavelength of $664 \mathrm{~nm}$. The reflectance spectra of each muskmelon seed were calculated by averaging the reflectance value of all the pixel points within the extracted contour. The results were shown in Figure 4a, where obvious difference could be observed for the trends of the hyperspectral curves of different varieties of muskmelon seeds. In addition, noise could be observed within the wavelength range of 400-450 nm, which was caused by the low responsibility of the CCD and low light source intensity at this wavelength range. In order to ensure the reliability of the data, only reflectance data within the spectral range of 450-720 nm were included for discriminative classifier modeling, as was shown in Figure 4b.



Figure 3 Snake contour segmentation of muskmelon seeds at the wavelength of $664 \mathrm{~nm}$ 


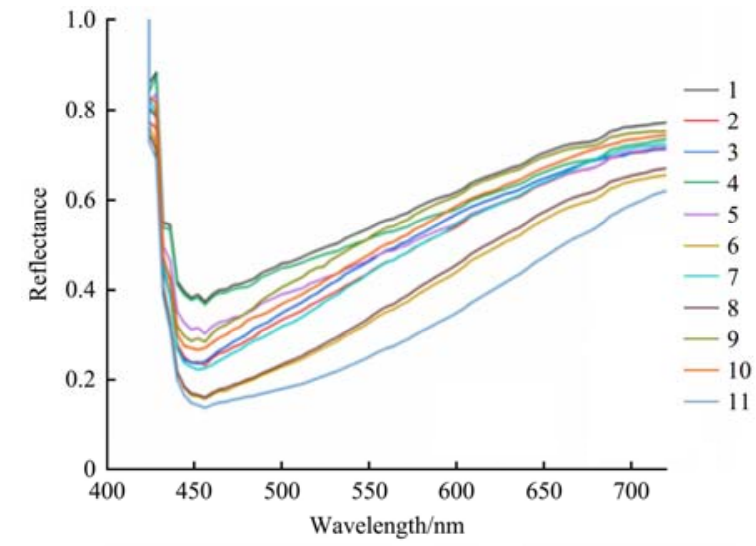

a. Reflectance of each sample. Number 1 to 11 correspond to: bairoushabai, baiyu2000, hongbaoshiyang, hongroushabai, lvbaoshiyang, meihua, meiqi, naixiangmiwang, kaqihuanghou, langchaoA710, and sumilong

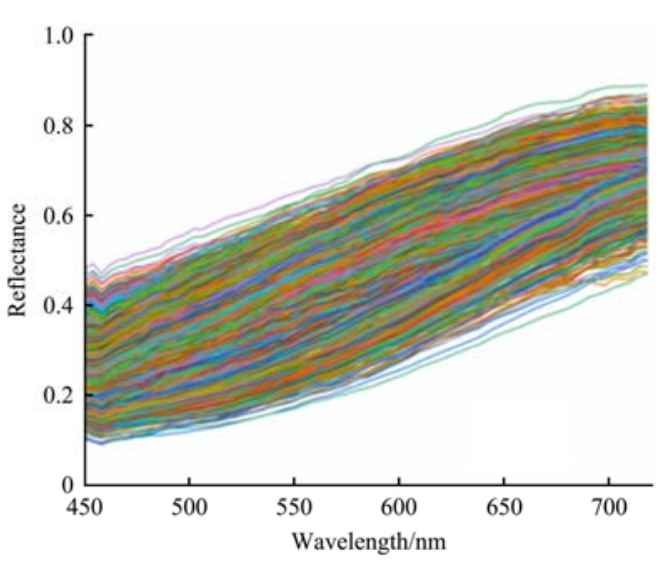

b. All the reflectance spectra of 11 varieties of muskmelon seeds

Figure 4 Reflectance spectra of muskmelon seed

\subsection{Data preprocessing analysis}

SG smoothing algorithm and MSC algorithm were first employed to preprocess the spectra to reduce the influence of irrelevant information and highlight the effective information of the spectrum. The results of data preprocessing were shown in Figure 5. It was observed from Figure $5 a$ that the reflectance spectra preprocessed by SG smoothing were smoother than the original spectra in Figure 4b, indicating parts of the noise were eliminated. Figure $5 \mathrm{~b}$ demonstrated that reflectance spectra preprocessed by MSC were more concentrated than the original spectra. In addition, large differences occurred within the wavelength range of $450-500 \mathrm{~nm}, 525-650 \mathrm{~nm}$, and $675-700 \mathrm{~nm}$ for the reflectance spectra of muskmelon seed varieties.

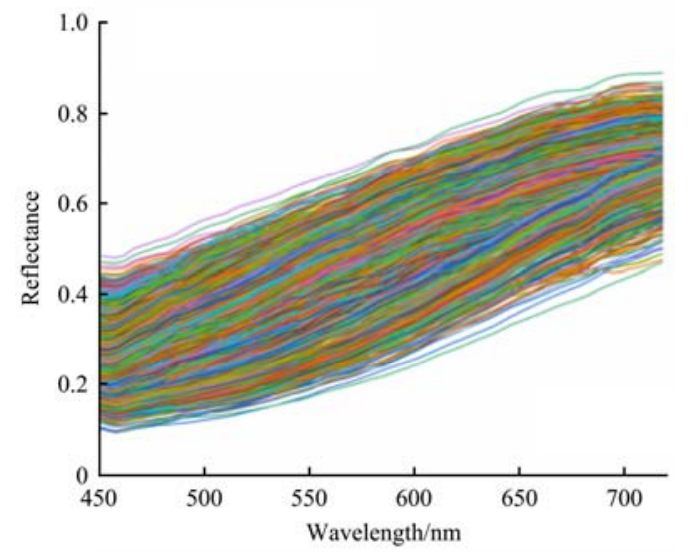

a. SG preprocess



b. MSC preprocess

Figure 5 Spectral curves of all samples were preprocessed by SG smoothing and MSC

\subsection{Results of discrimination}

To construct discriminating model, eleven varieties of muskmelon seeds "bairoushabai”, "baiyu2000”, "hongbaoshiyang”, "hongroushabai", "Ivbaoshiyang”, "meihua”, "meiqi”, "naixiangmiwang”, "kaqihuanghou”, "langchaoA710”, and "sumilong" were marked as 1-11 respectively. SG smoothing algorithm and MSC algorithm were first employed to preprocess the spectra of the muskmelon seeds. PCA and SPA were then used to extract the spectral feature of both the preprocessed spectra and original spectra. After that, discriminating models based on SVM, LDA and CNN were respectively established to classify the muskmelon seeds. The structure of CNN was shown in Figure 6 . The results obtained by these models were shown in Table 1, Table 2 and Table 3. In these tables, $\mathrm{N}_{\mathrm{p}}$ denotes the number of characteristic wavelengths or principal components.

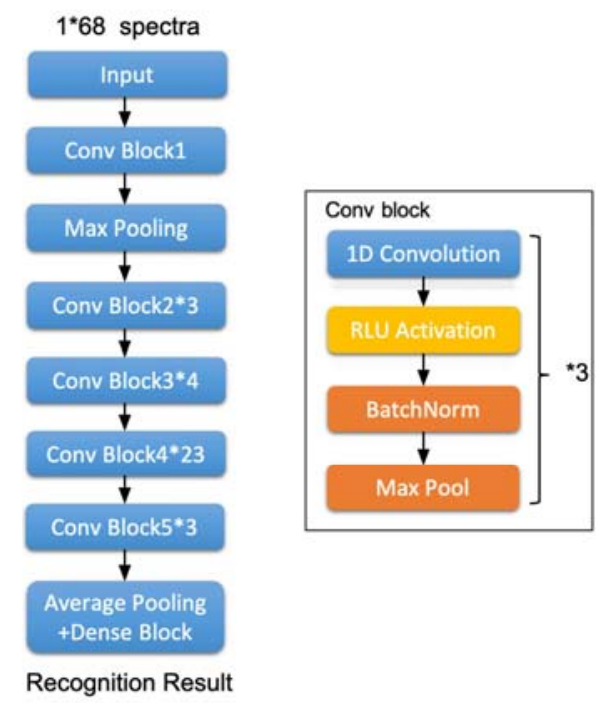

Figure 6 Structure of ResNet-101 convolutional neural networks

3.3.1 Optimal discrimination accuracy for the models

It was observed that the discrimination accuracy of LDA model based on full-wavelength data was the highest. More specifically, the discrimination accuracy for both the training set and the test set reached a high value of $100 \%$ whether the spectral data was preprocessed by SG smoothing/MSC algorithm or not (see Table 1-3). As far as the discriminating model based on SVM was concerned, the discriminating accuracy for the training set and testing set was as relatively low as $99.3 \%$ and $96.15 \%$ (see Table 1). The discriminating accuracy can be improved to a high 
value of $99.23 \%$ for the test set when the MSC algorithm was employed to preprocess the reflectance data, as was shown in Table 3. The value of the discriminating accuracy approached that for the LDA model.

Table 1 Discriminating results of muskmelon seed variety without preprocessing

\begin{tabular}{ccccc}
\hline $\begin{array}{c}\text { Spectral Feature } \\
\text { Extraction }\end{array}$ & $\mathrm{N}_{\mathrm{p}}$ & $\begin{array}{c}\text { Classification } \\
\text { algorithm }\end{array}$ & $\begin{array}{c}\text { Accuracy for } \\
\text { train set/\% }\end{array}$ & $\begin{array}{c}\text { Accuracy for } \\
\text { test set/\% }\end{array}$ \\
\hline Full & 67 & SVM & 99.33 & 96.15 \\
SPA & 26 & SVM & 97.57 & 94.61 \\
PCA & 9 & SVM & 93.72 & 91.28 \\
Full & 67 & LDA & 100 & 100 \\
SPA & 26 & LDA & 99.00 & 98.00 \\
PCA & 9 & LDA & 92.00 & 91.00 \\
Full & 67 & CNN & 90.33 & 81.03 \\
SPA & 26 & CNN & 90.33 & 76.92 \\
PCA & 9 & CNN & 90.33 & 66.41 \\
\hline
\end{tabular}

Table 2 Discriminating results of muskmelon seed variety with SG smooth preprocessing

\begin{tabular}{ccccc}
\hline $\begin{array}{c}\text { Spectral Feature } \\
\text { Extraction }\end{array}$ & $\mathrm{N}_{\mathrm{p}}$ & $\begin{array}{c}\text { Classification } \\
\text { algorithm }\end{array}$ & $\begin{array}{c}\text { Accuracy for } \\
\text { train set/\% }\end{array}$ & $\begin{array}{c}\text { Accuracy for } \\
\text { test set/\% }\end{array}$ \\
\hline Full & 67 & SVM & 99.22 & 96.15 \\
SPA & 30 & SVM & 98.34 & 95.38 \\
PCA & 8 & SVM & 92.51 & 90.00 \\
Full & 67 & LDA & 100 & 100 \\
SPA & 30 & LDA & 98.00 & 98.00 \\
PCA & 8 & LDA & 92.00 & 90.00 \\
Full & 67 & CNN & 90.33 & 81.03 \\
SPA & 30 & CNN & 90.33 & 76.92 \\
PCA & 8 & CNN & 90.33 & 66.41 \\
\hline
\end{tabular}

Table 3 Discriminating results of muskmelon seed variety with MSC preprocessing

\begin{tabular}{ccccc}
\hline $\begin{array}{c}\text { Spectral Feature } \\
\text { Extraction }\end{array}$ & $\mathrm{N}_{\mathrm{p}}$ & $\begin{array}{c}\text { Classification } \\
\text { algorithm }\end{array}$ & $\begin{array}{c}\text { Accuracy for } \\
\text { train set/\% }\end{array}$ & $\begin{array}{c}\text { Accuracy for } \\
\text { test set/\% }\end{array}$ \\
\hline Full & 67 & SVM & 99.88 & 99.23 \\
SPA & 27 & SVM & 99.22 & 97.69 \\
PCA & 29 & SVM & 99.88 & 99.23 \\
Full & 67 & LDA & 100 & 100 \\
SPA & 27 & LDA & 99.00 & 99.00 \\
PCA & 29 & LDA & 99.00 & 98.00 \\
Full & 67 & CNN & 90.22 & 86.67 \\
SPA & 27 & CNN & 90.22 & 62.50 \\
PCA & 29 & CNN & 90.33 & 85.64 \\
\hline
\end{tabular}

But for the discriminating model based on CNN, the discriminating accuracy was much lower than the LDA model and the SVM model. For the CNN model based on the full-wavelength data without preprocessing, discrimination accuracy was only $81.03 \%$ for the test set (Table 1). A relatively high accuracy of $86.67 \%$ can be achieved after MSC algorithm was applied to preprocessing the data (Table 3). This value, however, was still much lower than that achieved by the LDA model and the SVM model. These findings indicated that the performance of the CNN discriminating model was inferior to that of the SVM model and LDA model, which showed that CNN had no advantage for small sample.

3.3.2 Effects of preprocessing

By comparing Table 1 and Table 3, it can be seen that the preprocessing method of MSC can effectively improve the accuracy of the discriminating models. The SG smoothing pretreatment, on the contrary, did not improve the discrimination accuracy and even leads to slight reduction in the accuracy in some cases. The reduction maybe resulted from the fact that some features of the spectrum disappear in the smoothing process.

To further understand the different effects of the MSC and the SG smoothing pretreatment, T-distributed stochastic neighbor embedding (t-SNE) was used to project the data into 2-dimensional space for visualization and the results were shown in Figure 7. The distribution of muskmelon seed varieties in Figure 7a was similar to that in Figure 7b, which indicated that preprocessing by SG smoothing did not improve the distinguishability of the data. It confirmed the conclusion that SG smoothing pretreatment did not improve the discrimination accuracy of the models.
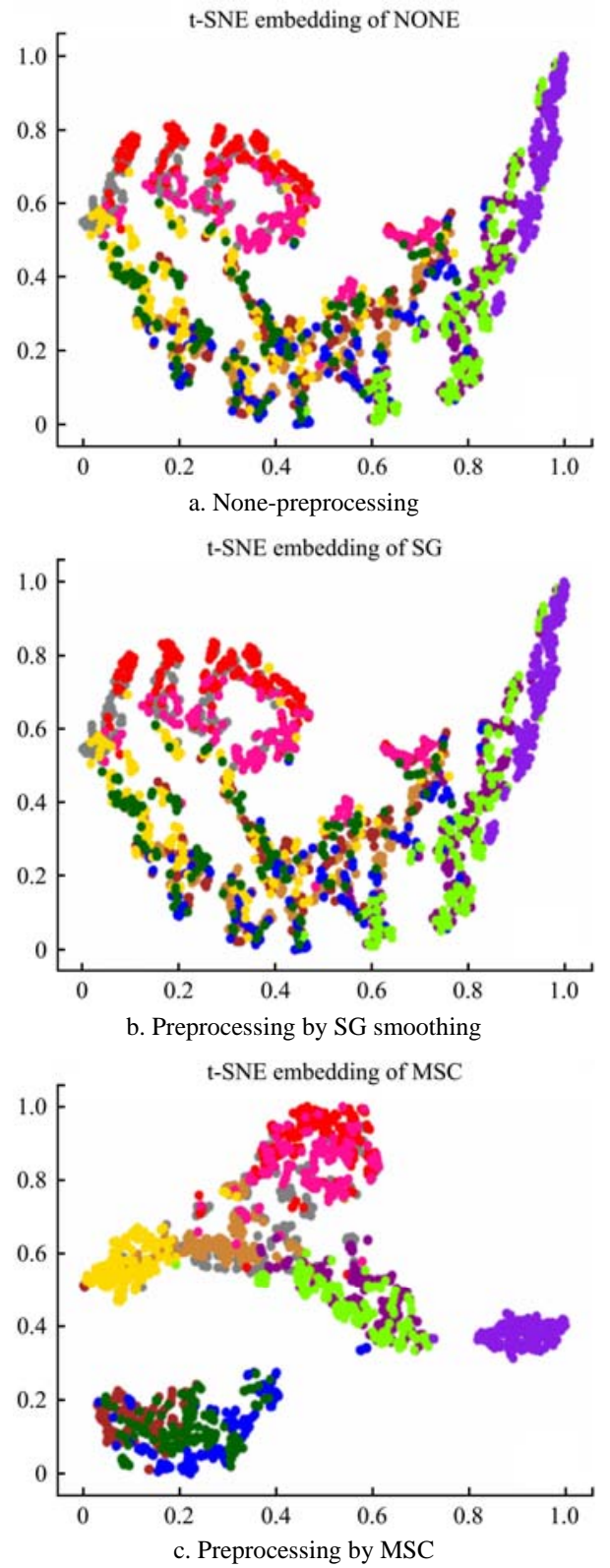

Figure 7 T-SNE visualization based on different data preprocessing

But for MSC preprocessing, the distribution of the data preprocessed by MSC (Figure 7c) was obviously different from that of the original data (Figure 7a). Parts of the seed variety were separated in the 2-dimensional space, as was shown in Figure 7c. These findings demonstrated that MSC pretreatment can improve 
the distinguishability of the data. Subsequently, the classification accuracy of the discriminating models can be improved.

\subsubsection{Effects of spectral feature extraction}

To examine whether these discriminating models can achieve high accuracy with less spectral features, PCA and SPA were employed to extract spectral features and their impacts on the models were shown in Table 1-3. For the discriminating models based on the original data (reflectance data were not preprocessed by SG and MSC, see Table 1), the use of both PCA and SPA to extract spectral feature usually leaded to reduction in the accuracy. For example, the discriminating accuracy of the SVM model based on full-wavelength data was $96.15 \%$ for the test set. The value decreased to $94.64 \%$ when SPA was used to select the characteristic wavelength and it further decreases to $91.28 \%$ when PCA was used. The decreasing trend can also be observed for both LDA and CNN discriminating models. For the discriminating models based on the spectral data preprocessed by SG smoothing (see Table 2), similar results were achieved since the SG smoothing did not improve the distinguishability of the data.

However, for the the discriminating models based on the spectral data which were preprocessed by MSC, the case was different (Table 3). The discriminating accuracy of the test set for the SVM model remained at a high value of $99.23 \%$ when PCA was applied for feature extraction. This value of accuracy was the same with that of the model based on full-wavelength data even when the number of the spectral feature is reduced from 67 to 27 . In addition, the discrimination accuracy of the test set for the LDA model was as high as $99.00 \%$ when SPA was used to extract 27 characteristic wavelengths from 67. These findings demonstrated that the SVM/LDA discriminating models combined with MSC preprocessing and PCA/SPA feature extracting was a suitable approach to achieve relatively high accuracy with less spectral features.

\section{Conclusion}

LCTF-based hyperspectral imaging technology combined with machine learning was proposed in this paper to identify muskmelon seed varieties. With the reflectance data acquired by LCTF-based hyperspectral imaging technology, discriminating models based on SVM, LDA and CNN were established to classify 11 varieties of muskmelon seeds. The effects of the data preprocessing and the spectral feature extracting on the discriminating accuracy were also investigated. It was revealed that the discrimination accuracy of the training set and test set for the LDA model reached the highest value of $100 \%$. The discrimination results of the training set and test set of the SVM discriminating model can reach $99.88 \%$ and $99.23 \%$ when the spectrum data were preprocessed by MSC. It is also demonstrated that the SVM/LDA discriminating model combined with MSC preprocessing and PCA/SPA feature extracting was a suitable approach to achieve relatively high accuracy with less spectral features. The findings in this paper indicate that LCTF-based hyperspectral imaging technology combined with machine learning is a feasible approach to identify the varieties of muskmelon seeds.

\section{Acknowledgments}

We deeply thank for the Key-Area Research and Development Program of Guangdong Province (No.2019B020219002 and
2019B020214005), Guangdong Provincial Rural strategic revitalization project in 2019 (YCN (2019) No. 73).

\section{[References]}

[1] Thakur H, Sharma S, Thakur M. Recent trends in muskmelon (Cucumis melo L.) research: an overview. Journal of Horticultural Science and Biotechnology, 2019; 94(4): 533-547. doi: 10.1080/ 14620316.2018.1561214.

[2] Venora G, Grillo O, Shahin M A, et al. Identification of Sicilian landraces and Canadian cultivars of lentil using an image analysis system, Food Research International, 2007; 40(1): 161-166. doi: 10.1016/ j.foodres.2006.09.001.

[3] Huebner F R, Hussain A, Lookhart G L, et al. Discrimination of sister-line IR rice varieties by polyacrylamide gel electrophoresis and reversed-phase high-performance liquid chromatography. Cereal Chemistry, 1991; 68(6): 583-588.

[4] Wu J, Yu H C, Dai H F, et al. Metabolite profiles of rice cultivars containing bacterial blight-resistant genes are distinctive from susceptible rice. Acta Biochimica et Biophysica Sinica, 2012; 44(8), 650-659. doi: 10.1093/abbs/gms043.

[5] Liu X, Wang Y, Su Q, Wang Z. Study On Identification System Of Maize Seeds varieties Based On Machine Vision, Computer and Computing Technologies in Agriculture, 2009; 3: 2207-2216. doi:10.1007/ 978-1-4419-0213-9_72

[6] Deepak Kumar Jain, et al. An approach for hyperspectral image classification by optimizing SVM using self organizing map, Journal of Computational Science, 2018; 25: 252-259. doi: 10.1016/ j.jocs.2017.07.016

[7] Li C, Jiang K, Feng Q, Wang X, Meng Z, Wang S, Gao Y. Identification of melon seed varieties based on chlorophyll fluorescence and reflectance spectra. Spectroscopy and Spectral Analysis, 2018; 1: 151-156. doi: 10.3964/j.issn.1000-0593(2018)01-0151-06 (in Chinese)

[8] Guo P T, Li M F, Luo W, et al. Estimation of foliar nitrogen of rubber trees using hyperspectral reflectance with feature bands. Infrared Physics \& Technology, 2019; 102: 103021. doi: 10.1016/j.infrared.2019.103021.

[9] Zhang X L, Liu F, Nie P C, et al. Rapid Detection of Nitrogen Content and Distribution in Oilseed Rape Leaves Based on Hyperspectral Imaging, Spectroscopy and Spectral Analysis, 2014; 34(9): 2513-2518. doi: 10.3964/j.issn.1000-0593(2014)09-2513-06. (in Chinese)

[10] Barbedo J G A. Detection of nutrition deficiencies in plants using proximal images and machine learning: A review, Computers and Electronics in Agriculture, 2019; 162: 482-492. doi: 10.1016/ j.compag.2019.04.035

[11] Gutierrez S, Wendel A, Underwood J. Ground based hyperspectral imaging for extensive mango yield estimation, Computers and Electronics in Agriculture, 2019, 157: 126-135. doi: 10.1016/j.compag.2018.12.041.

[12] Wendel A, Underwood J, Walsh K. Maturity estimation of mangoes using hyperspectral imaging from a ground based mobile platform, Computers and Electronics in Agriculture, 2018, 155: 298-313. doi: 10.1016/j.compag.2018.10.021.

[13] Li H Y, Jia S Y, Le Z C. Quantitative Analysis of Soil Total Nitrogen Using Hyperspectral Imaging Technology with Extreme Learning Machine, Sensors; 2019, 19(20): 4355 . doi: 10.3390/s19204355.

[14] Feng C H, Makino Y, Oshita S, et al. Hyperspectral imaging and multispectral imaging as the novel techniques for detecting defects in raw and processed meat products: Current state-of-the-art research advances, Food Control, 2018; 84: 165-176. doi: 10.1016/j.foodcont.2017.07.013.

[15] Ali M M, Bachik N A, Muhadi N A, et al. Non-destructive techniques of detecting plant diseases: A review, Physiological and Molecular Plant Pathology, 2019; 108: 101426. doi: 10.1016/j.pmpp.2019.101426.

[16] Zhang J C, Huang Y B, Pu R L, et al. Monitoring plant diseases and pests through remote sensing technology: A review, Computers and Electronics in Agriculture, 2019; 165: 104943. doi: 10.1016/j.compag. 2019.104943.

[17] Sun J, Cong S L, Mao H P, et al. Quantitative detection of mixed pesticide residue of lettuce leaves based on hyperspectral technique, Journal of Food Process Engineering, 2018; 41: e12654. doi: 10.1111/ jfpe.12654.

[18] He X T, Feng X P, Sun D W, et al. Rapid and Nondestructive Measurement of Rice Seed Vitality of Different Years Using Near-Infrared Hyperspectral Imaging, Molecules, 2019; 24(12): 2227. doi: 10.3390/ 
molecules24122227.

[19] Zhu Hongyun, Wang Changlong, Wang Jianbin, Ma Xiaolin. Signal denoising method based on singular value decomposition and Savitzky-Golay filter. Computer Applications, 2015, 35(10): 3004-3007, 3012. doi: 10.11772/j.issn.1001-9081.2015.10.3004(in Chinese)

[20] Nie P C, Zhang J N, Feng X P, et al. Classification of hybrid seeds using near-infrared hyperspectral imaging technology combined with deep learning, Sensors and Actuators B: Chemical, 2019, 296: 126630. doi: 10.1016/j.snb.2019.126630.

[21] Feng L, Zhu S S, Zhang C, et al. Identification of Maize Kernel Vigor under Different Accelerated Aging Times Using Hyperspectral Imaging, Molecules, 2018; 23(12): 3078. doi: 10.3390/molecules23123078.

[22] Zhang J, Dai L M, Cheng F. Classification of Frozen Corn Seeds Using Hyperspectral VIS/NIR Reflectance Imaging, Molecules, 2019; 24(1): 149. doi: $10.3390 /$ molecules 24010149 .

[23] Qiu Z J, Chen J, Zhao Y Y, et al. Variety Identification of Single Rice Seed Using Hyperspectral Imaging Combined with Convolutional Neural Network, Applied sciences-Basel, 2018, 8(2): 212. doi: 10.3390/ app8020212.

[24] Baek I, Kim M S, Cho B K, et al. Selection of Optimal Hyperspectral Wavebands for Detection of Discolored, Diseased Rice Seeds, Applied Sciences-Basel, 2019; 9(5): 1027. doi: 10.3390/app9051027.

[25] Zhao Y Y, Zhu S S, Zhang C, et al. Application of hyperspectral imaging and chemometrics for variety classification of maize seeds, RSC Advances, 2018; 8(3): 1337-1345. doi: 10.1039/c7ra05954j.

[26] Yang Q S, Marchant J A. Accurate blemish detection with active contour models, Computers and Electronics in Agriculture, 1996; 14(1): 77-89. doi: 10.1016/0168-1699(95)00038-0. 